\title{
The Cooling Flow to Accretion Flow Transition
}

\section{Citation}

Quataert, Eliot, and Ramesh Narayan. 2000. “The Cooling Flow to Accretion Flow Transition.” The Astrophysical Journal 528 (1): 236-42. https://doi.org/10.1086/308171.

\section{Permanent link}

http://nrs.harvard.edu/urn-3:HUL.InstRepos:41384930

\section{Terms of Use}

This article was downloaded from Harvard University's DASH repository, and is made available under the terms and conditions applicable to Other Posted Material, as set forth at http:// nrs.harvard.edu/urn-3:HUL.InstRepos:dash.current.terms-of-use\#LAA

\section{Share Your Story}

The Harvard community has made this article openly available.

Please share how this access benefits you. Submit a story.

Accessibility 


\title{
THE COOLING FLOW TO ACCRETION FLOW TRANSITION
}

\author{
Eliot Quataert AND RAMESh NARAYAN \\ Harvard-Smithsonian Center for Astrophysics, 60 Garden St., Cambridge, MA 02138; equataert, rnarayan, @cfa.harvard.edu
}

Draft version August 20, 2018

\begin{abstract}
Cooling flows in galaxy clusters and isolated elliptical galaxies are a source of mass for fueling accretion onto a central supermassive black hole. We calculate the dynamics of accreting matter in the combined gravitational potential of a host galaxy and a central black hole assuming a steady state, spherically symmetric flow (i.e., no angular momentum). The global dynamics depends primarily on the accretion rate. For large accretion rates, no simple, smooth transition between a cooling flow and an accretion flow is possible; the gas cools towards zero temperature just inside its sonic radius, which lies well outside the region where the gravitational influence of the central black hole is important. For accretion rates below a critical value, however, the accreting gas evolves smoothly from a radiatively driven cooling flow at large radii to a nearly adiabatic (Bondi) flow at small radii. We argue that this is the relevant parameter regime for most observed cooling flows. The transition from the cooling flow to the accretion flow should be observable in M87 with the Chandra X-ray Observatory.
\end{abstract}

Subject Headings: galaxies: cooling flows - accretion, accretion disks - galaxies: individual (M87)

\section{INTRODUCTION}

X-ray observations of clusters of galaxies and isolated elliptical galaxies indicate the presence of significant quantities of hot gas whose radiative cooling time is much less than a Hubble time (see, e.g., Fabian 1994 for a review). This is generally interpreted as evidence for a cooling flow, i.e., the radiatively driven accretion of gas onto a central cluster galaxy or towards the center of the host galaxy (e.g., Cowie \& Binney 1977; Fabian \& Nulsen 1977; Mathews \& Bregman 1978). The inferred accretion rates range from $\sim 0.1 M_{\odot} \mathrm{yr}^{-1}$ to $\sim 10^{3} M_{\odot} \mathrm{yr}^{-1}$ and are often inferred to decrease substantially with decreasing radius, roughly as $\dot{M} \propto r$ (e.g., Stewart et al. 1984). The decreasing $\dot{M}$ is probably evidence for an inhomogeneous flow, in which some of the gas (that which is denser) cools out of the hot flow at large radii.

It has long been recognized that a cooling flow may be an important source of fuel for accretion onto a central supermassive black hole in the host galaxy (e.g., Bailey 1980, Nulsen, Stewart, \& Fabian 1984). The goal of this paper is to calculate in some detail the dynamics of accreting matter in the central regions of a galaxy which harbors both a cooling flow and a supermassive black hole. We restrict ourselves to spherically symmetric flows.

Our model and its assumptions are discussed in the next section ( $(2)$. In $\S 3$ we present solutions to our model problem and explain the physics which governs the dynamical transition from a cooling flow to an accretion flow. Finally, $\S 4$ sums up and discusses the observational implications of our work.

\section{THE MODEL}

We consider a simplified model which we believe captures much - although not all $(\S 4)$ - of the interesting physics of the transition from a cooling flow to an accretion flow. The primary assumptions of our model are: (1) steady state and (2) spherical symmetry. Aside from adiabatic compression, we assume negligible heating of the gas, and we approximate its cooling as due to bremsstrahlung emission.

Cooling flows are inferred not to conserve mass. Theoretically, this can be understood by noting that the cooling time of the gas is comparable to the inflow time. Overdense "blobs" can therefore condense out of the flow before accreting. We model this by including a mass drop out term in the continuity equation (cf White \& Sarazin 1987):

$$
\frac{d \dot{M}}{d r}=q \frac{\dot{M}}{r} \frac{t_{\text {inf }}}{t_{\text {cool }}}, \quad \dot{M}=-4 \pi r^{2} \rho v .
$$

Momentum and energy conservation are given by

$$
v \frac{d v}{d r}=-\frac{d \phi}{d r}-\frac{1}{\rho} \frac{d \rho c_{s}^{2}}{d r}
$$

and

$$
\rho v T \frac{d s}{d r}=\frac{\rho v}{\gamma-1} \frac{d c_{s}^{2}}{d r}-c_{s}^{2} v \frac{d \rho}{d r}=-B \rho^{2} c_{s} .
$$

The quantities $\dot{M}, \rho, v, \phi, c_{s}, T, s$, and $\gamma$ represent the mass accretion/inflow rate, the mass density, the radial velocity (negative inwards), the gravitational potential, the isothermal sound speed, the temperature, the entropy per unit mass, and the gas adiabatic index, respectively. The quantity $B \approx 5.6 \times 10^{16} \mathrm{ergs} \mathrm{cm}^{2} \mathrm{~g}^{-2}$ is the normalization for the bremsstrahlung emission.

The efficiency of mass drop out depends on the ratio of the inflow time of the gas $\left(t_{\text {inf }} \equiv r /|v|\right)$ to the cooling time $\left(t_{\text {cool }} \equiv c_{s} / B \rho\right)$. When $t_{\text {inf }} \sim t_{\text {cool }}$ (as in a cooling flow), mass drop out can be important since there is time for overdense regions to condense out. When $t_{\text {inf }} \ll t_{\text {cool }}$, 
drop out is negligible since the gas accretes before cooling. The parameter $q$ in equation (11) normalizes the efficiency of mass drop out; we expect $q \sim 1$.

We take the gravitational potential to be given by

$$
\phi=\frac{-G M}{r-r_{g}}+\sigma^{2} \ln r \text { for } r>r_{b}
$$

and

$$
\phi=\frac{-G M}{r-r_{g}}-\sigma^{2}\left(\frac{r}{r_{b}}\right)^{-\beta+1} \text { for } r<r_{b}
$$

The left most term in equations (4) and (5) is the gravitational potential due to a black hole of mass $M$ and Schwarzschild radius $r_{g}=2 G M / c^{2}$; the $1 /\left(r-r_{g}\right)$ mimics the effects of General Relativity (Paczyński \& Wiita 1980).

$H S T$ observations of the centers of elliptical galaxies indicate that those which harbor massive black holes have central surface brightness "cores." The surface brightness rises steeply with decreasing radius at large radii, but flattens out in the inner portions of the galaxy (e.g., Lauer et al. 1995; Faber et al. 1995; Kormendy \& Richstone 1995).1. Our mass model for the galaxy in equations (4) and (5) is intended to reflect these observations. Outside a break radius $r_{b}$, the galaxy has a constant velocity dispersion $(\sigma)$ while inside that radius we assume that light traces mass (aside from the black hole) and so the enclosed mass profile flattens $(\beta<1)$ in accord with the flattening surface brightness.

Typical observed values of $\beta$ and $r_{b}$ are $\approx 0.25$ and $\approx 1$ kpc, respectively (Faber et al. 1995); we use such values in our numerical results of $\S 3.1$. For analytical estimates, however, we take $r_{b} \rightarrow 0$, i.e., we model the galaxy as having a constant velocity dispersion everywhere.

The gravitational potential is predominantly that of the galaxy for $r \gtrsim r_{\mathrm{tr}}$ while it is predominantly that of the black hole for $r \lesssim r_{\text {tr }}$, where the transition radius is (for $\left.r_{b} \rightarrow 0\right)$

$$
r_{\mathrm{tr}} \approx \frac{G M}{\sigma^{2}} \approx 0.05 \mathrm{kpc}\left(\frac{M}{10^{9} M_{\odot}}\right)\left(\frac{\sigma}{300 \mathrm{~km} \mathrm{~s}^{-1}}\right)^{-2} .
$$

For a galaxy with $\sigma \approx 300 \mathrm{~km} \mathrm{~s}^{-1}$ in the Virgo cluster, a distance $\approx 20 \mathrm{Mpc}$ away, $r_{\text {tr }}$ corresponds to $\approx 0.5$ arcsec. This is comparable to the angular resolution of the Chandra X-ray Observatory (CXO), indicating that the presence of a central black hole in nearby ellipticals may have observable effects on the cooling flow X-ray emission (see $\S 4.1$ ).

\section{MODEL SOLUTIONS}

We are interested in solutions to our model problem which undergo a subsonic to supersonic transition at a sonic radius $r_{s}$. Rewriting equations (11)-(3) yields

$$
v \frac{d v}{d r}=\frac{N}{D}
$$

where

$$
N=\frac{-d \phi}{d r}+\frac{2 \gamma c_{s}^{2}}{r}+\frac{B \rho c_{s}(\gamma-1+\gamma q)}{v}
$$

and

$$
D=1-\frac{\gamma c_{s}^{2}}{v^{2}}
$$

Since $D=0$ at the sonic point, we must have $N=0$ for a smooth transition.

We take our outer boundary conditions to be specified values of $\rho_{o}$ and $c_{o}$, the density and sound speed, at an outer radius $r_{\mathrm{o}}$. Equations (11)-(3) have two eigenvalues, taken here to be the sonic radius, $r_{s}$, and the accretion rate at the sonic radius, $\dot{M}\left(r_{s}\right)$. We find our solutions by shooting out from $r_{s}$ and adjusting $\dot{M}\left(r_{s}\right)$ and $r_{s}$ to satisfy the outer boundary conditions.

For most of this paper we scale our models to observations of M87: $r_{\mathrm{o}} \approx 100 \mathrm{kpc}, \rho_{o} \approx 10^{-27} \mathrm{~g} \mathrm{~cm}^{-3}$, $\sigma \approx 300 \mathrm{~km} \mathrm{~s}^{-1}, c_{o} \approx \sigma$ (e.g., Stewart et al. 1984) and $M \approx 3 \times 10^{9} M_{\odot}$ (Harms et al. 1994; Ford et al. 1994; Macchetto et al. 1997). In M87, $\dot{M}$ is inferred to decrease from $\approx 10 M_{\odot} \mathrm{yr}^{-1}$ at $\approx 70 \mathrm{kpc}$ to $\lesssim 1 M_{\odot} \mathrm{yr}^{-1}$ at a few kilo-parsecs (Stewart et al. 1984). As shown below, this is reasonably well captured by a $q=0.6$ model.

Although strictly an eigenvalue of our problem, an excellent estimate of the value of $\dot{M}$ at the outer boundary, $\dot{M}\left(r_{\mathrm{o}}\right) \equiv \dot{M}_{o}$, can be made by imposing the requirement that the gas be a cooling flow at $r_{\mathrm{o}}$. This requires that the inflow time of the gas be comparable to the local radiative cooling time which in turn requires $v\left(r_{\mathrm{o}}\right) \equiv v_{o} \approx-B \rho_{o} r_{o} / c_{o}$ and therefore (cf Fabian \& Nulsen 1977)

$$
\begin{aligned}
\dot{M}_{o} & \approx \frac{4 \pi B \rho_{o}^{2} r_{o}^{3}}{c_{o}} \\
& \approx 13 M_{\odot} \mathrm{yr}^{-1}\left(\frac{\rho_{o}}{10^{-27} \mathrm{~g} \mathrm{~cm}^{-3}}\right)^{2} \\
& \times\left(\frac{r_{o}}{100 \mathrm{kpc}}\right)^{3}\left(\frac{c_{o}}{300 \mathrm{~km} \mathrm{~s}^{-1}}\right)^{-1} .
\end{aligned}
$$

The primary question is what happens to matter accreting at this rate at smaller radii.

In the absence of a central point mass a cooling flow with $\dot{M}_{o}$ given by equation (10) would have the following structure:

$c_{s} \approx \sigma, \dot{M} \propto r^{3 q /(q+2)}, \rho \propto r^{-3 /(q+2)}, \quad \& v \propto r^{(q-1) /(q+2)}$.

This is readily verified by substitution into equations (1)(3) under the assumption that the gas is subsonic and that the potential is logarithmic $\left(M=0, r_{b} \rightarrow 0\right)$. Note, however, that if $q<1$ the above scalings show that the Mach number of the flow increases as the radius decreases. At $r_{o}$, the Mach number is

$$
\begin{aligned}
\frac{v_{o}}{c_{o}} & \approx 0.02\left(\frac{\rho_{o}}{10^{-27} \mathrm{~g} \mathrm{~cm}^{-3}}\right)\left(\frac{r_{o}}{100 \mathrm{kpc}}\right)\left(\frac{c_{o}}{300 \mathrm{~km} \mathrm{~s}^{-1}}\right)^{-2} \\
& \approx 0.02\left(\frac{\dot{M}}{13 M_{\odot} \mathrm{yr}^{-1}}\right)^{1 / 2}\left(\frac{r_{o}}{100 \mathrm{kpc}}\right)^{-1 / 2} \\
& \times\left(\frac{c_{o}}{300 \mathrm{~km} \mathrm{~s}^{-1}}\right)^{-3 / 2} .
\end{aligned}
$$

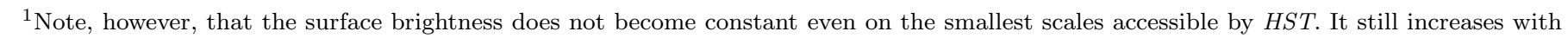
decreasing radius, but with a noticeably smaller power law slope. 
Therefore, there is a critical radius at which the flow would make a sonic transition:

$$
r \equiv r_{\text {crit }} \approx r_{\mathrm{o}}\left(v_{o} / c_{o}\right)^{(q+2) /(1-q) .}
$$

The key physics which determines the nature of the cooling flow to accretion flow transition is whether $r_{\text {tr }} \gtrsim r_{\text {crit }}$ or $r_{\text {tr }} \lesssim r_{\text {crit }}$. That is, is the radius where the gravitational influence of the black hole becomes important inside or outside of the radius where, in the absence of the point mass, the cooling flow would have its sonic point?

If $r_{\text {tr }} \lesssim r_{\text {crit }}$ the cooling flow is oblivious to the existence of the point mass. It undergoes its sonic transition with little modification because of the black hole. Inside of the sonic point, the gas quickly cools towards zero temperature. The reason is that in the supersonic zone a cooling flow in a logarithmic potential has $v \propto\left[\ln \left(r_{s} / r\right)\right]^{1 / 2}$ and $\rho \propto r^{-2} v^{-1} \sim r^{-2}$. The velocity (density) therefore rises more slowly (quickly) with decreasing radius than in the subsonic zone, where the cooling time was of order the inflow time. Consequently, in the supersonic zone the cooling time becomes much shorter than the inflow time and the gas cools rapidly (see, e.g., Fig. 4 of Sarazin \& White 1987). In this case, a simple cooling flow to accretion flow transition is not possible. Whether such a cooling flow is an important source of mass for an accretion flow depends critically on the fate of the rapidly cooling matter inside the sonic point (e.g., does it form stars which lose mass to feed the central black hole?).

For M87, $r_{\mathrm{tr}} \approx 0.15 \mathrm{kpc}$; this is comparable to $r_{\text {crit }}$ if $q=0$. Many other observed cooling flows have $\dot{M}_{o} \gg$ $10 M_{\odot} \mathrm{yr}^{-1}$ (M87's value) and would have $r_{\text {crit }} \gg r_{\text {tr }}$ if $q$ were equal to 0 . Significant mass drop out is, however, generally inferred in such cooling flows (for M87, $q \approx 0.6$ is appropriate; see below). From equation (11) it follows that if $q \sim 1$ the Mach number of the cooling flow is roughly constant. This insures that the gas remains subsonic down to the radius where the potential of the black hole begins to dominate the potential of the galaxy (i.e., $r_{\mathrm{tr}} \gtrsim r_{\text {crit }}$ ). We now turn our attention to this regime.

There is one conceptual complication in understanding the cooling flow to accretion flow structure in the regime where $r_{\text {tr }} \gtrsim r_{\text {crit }}$. Namely, in the point mass potential (i.e., for $r \ll r_{\text {tr }}$ ), there are two viable analytical solutions to equations (1)-(3). One is Bondi's adiabatic spherical accretion solution (Bondi 1952). The other (valid only for $\gamma>11 / 7$ ) is a cooling flow solution (cf Fabian \& Nulsen 1977) which has $c_{s}^{2} \propto r^{-1}, \rho \propto r^{-7 / 4}$ and $v \propto r^{-1 / 4}$. It is not a priori obvious which of these solutions the cooling flow at large radii (in the galaxy's logarithmic potential) will match onto at small radii. One might in fact suspect, on the basis of "continuity" arguments, that the cooling flow at large radii would match onto a cooling flow at small radii.

Our calculations indicate, however, that this is not so. The reason is simply that there is no transonic cooling flow solution in the $r^{-1}$ potential. The Mach number of the cooling flow solution in a point mass potential decreases with decreasing radius as $r^{1 / 4}$; this solution cannot be matched to a sonic transition even with the introduction of a singularity into the point mass problem via the
$1 /\left(r-r_{g}\right)$ potential. 2 We have checked this carefully by numerically searching the $\dot{M}_{o}$ and $r_{s}$ space; we find that there is no transonic cooling flow solution for $r<r_{\mathrm{tr}}$.

The upshot of this analysis is that the cooling flow makes a smooth transition to a nearly adiabatic Bondi flow in the vicinity of $r_{\mathrm{tr}}$. This provides a direct dynamical link between the cooling flow and the accretion flow onto the central black hole.

The details of the flow structure near $r_{\mathrm{tr}}$ are shown in Figure 1, where we plot the temperature, density, Mach number, and the ratio of the cooling time to the inflow time for models with $q=0.2,0.6$, and 1 . Figure 2 shows the accretion rate as a function of radius for these models. The boundary conditions at $r_{\mathrm{o}}=100 \mathrm{kpc}$ are those appropriate for M87. In M87, $\dot{M}$ is inferred to decrease from $\approx 10 M_{\odot} \mathrm{yr}^{-1}$ at $\approx 70 \mathrm{kpc}$ to $\lesssim 1 M_{\odot} \mathrm{yr}^{-1}$ at a few kilo-parsecs (Stewart et al. 1984). This is reasonably well captured by our $q=0.6$ model. The additional values of $q$ are shown to indicate the range of expected behavior. Note that the models in Figures 1 and 2 assume that $r_{b}=0$, i.e., that the galaxy's potential is logarithmic everywhere. Modifications due to alternative potentials are discussed in $§ 3.1$.

The conceptually most important result in Figure 1 is the ratio of the cooling time to the inflow time. This is $\sim 1$ at large radii where the accreting matter is indeed a cooling flow but it increases rapidly at smaller radii as the flow undergoes the transition to nearly adiabatic (Bondi) accretion. Associated with this transition is a decrease in the importance of mass drop out. In the Bondi regime, $t_{\text {cool }} \gg t_{\text {inf }}$ and so overdense regions do not have time to condense out of the flow. This accounts for the nearly constant accretion rate for $r \lesssim 300$ pc in Figure 2 .

\subsection{The Importance of the Potential}

To illustrate the impact of the underlying potential of the host galaxy on the structure of the cooling flow, consider the analytical solution to equations (11)-(3) in the region where the galaxy's mass profile flattens (i.e., for $r_{\text {tr }} \lesssim r \lesssim r_{b}$ and $\left.\beta<1\right): c_{s}^{2} \propto r^{-\beta+1}, \rho \propto r^{-5 / 4-\beta / 4}$, and $v \propto r^{-3 / 4+\beta / 4}$ (for simplicity, we have taken $q=0$ ). Because the cooling flow is roughly virial, the gas temperature decreases inward. The Mach number of the resulting solution therefore increases rapidly inwards, as $r^{3 \beta / 4-5 / 4}$. For observed values of $\beta \approx 0.25$, the Mach number increases roughly as $r^{-1}$, much more rapidly than the $r^{-1 / 2}$ scaling in the logarithmic potential. One might worry that this would change the results of the previous section by forcing the cooling flow to have its sonic point outside of $r_{\text {tr }}$. Our calculations show, however, that this does not happen.

Figure 3 shows the temperature, density, Mach number and ratio of cooling time to inflow time for three models of M87 with $q=0.6$, and Figure 4 shows the corresponding predicted X-ray surface brightness profiles. The models in Figures 3 and 4 assume three different forms for the potential of the host galaxy: one purely logarithmic (solid line; the same model is also shown by a solid line in Figs. $1 \& 2$ ) and two with flattening mass profiles; $\beta \approx 0.25$ in-

\footnotetext{
${ }^{2}$ Since the Mach number of the cooling flow solution in the $1 / r$ potential decreases with decreasing radius the only way it could ever make a sonic transition is if it were forced to by a singularity in the potential (or by some other boundary condition at small radii).
} 
side $r_{b} \approx 0.7 \mathrm{kpc}$ (dotted line) or $r_{b} \approx 3 \mathrm{kpc}$ (dashed-dot line). 阝 $^{-2}$

As illustrated by Figures 3 and 4, the quantitative details of the flow structure depend (not surprisingly) on the form of the potential on kilo-parsec scales. The most important result of this subsection, however, is that even when the mass profile flattens, the cooling flow still has its sonic point well inside $r_{\text {tr }}$ and therefore still makes a smooth transition to a nearly adiabatic accretion flow. The qualitative picture outlined in the previous section is therefore unchanged. This is partially because the inferred Mach number in M87 at a few kilo-parsec is sufficiently small $(\approx 0.04$; see Fig. $1 \mathrm{c})$ that even if the Mach number increases linearly at small radii the black hole still becomes important before the cooling flow has its sonic point. In addition, because the temperature of the gas decreases somewhat if the galaxy's potential flattens, the black hole becomes important for the dynamics of the gas at larger radii (the transition radius, $r_{\mathrm{tr}}$, is effectively $\propto c_{s}^{-2}$ when $\left.c_{s}<\sigma\right)$. Note, for example, that $t_{\mathrm{cool}} / t_{\mathrm{inf}}$ is actually larger at smaller radii in the model with $r_{b}=3 \mathrm{kpc}$. This is because the decreasing temperature due to the flat mass profile implies that the flow undergoes its transition from a cooling flow to an accretion flow at a larger radius.

\section{DISCUSSION}

The theoretical picture which emerges from the above analysis is a very intuitive one: cooling flows in elliptical galaxies remain subsonic down to roughly kilo-parsec scales at which point they are gravitationally "captured" by the central supermassive black hole. They then undergo a transition to a nearly adiabatic Bondi flow and accrete onto the black hole. There is consequently a direct and simple dynamical transition from a cooling flow to an accretion flow.

Our calculations indicate that only if the cooling flow accretion rate on scales of a few kilo-parsecs is $\gtrsim 10-$ $100 M_{\odot} \mathrm{yr}^{-1}$ will the cooling flow have its sonic transition before being captured by the black hole (the precise $\dot{M}$ depends on the black hole mass - assumed $\gtrsim 10^{9} M_{\odot}$ - and the galaxy potential, i.e., $\beta, \sigma$, and $r_{b}$; less massive black holes, larger $\sigma$, and flatter galaxy mass profiles - larger $r_{b}$ and smaller $\beta$-require smaller $\dot{M}$ ). In this case, the gas cools rapidly just inside its sonic point, which lies outside the region where the gravitational influence of the black hole is important. Although $\dot{M} \gtrsim 10-100 M_{\odot} \mathrm{yr}^{-1}$ is readily achieved on $\sim 100 \mathrm{kpc}$ scales, mass drop out due to thermal instability, and the consequent decrease of $\dot{M}$ with decreasing radius, implies that observed cooling flows typically lie in the parameter regime where a smooth transition from a cooling flow to a nearly adiabatic accretion flow is the viable transonic solution. We find that this conclusion is valid even given uncertainties in the mass profile of the host galaxy on kilo-parsec scales (§3.1).

It is interesting to note that all of the solutions shown in Figures 1-5 have $r_{s} \ll r_{\text {tr }}$; that is, the sonic point lies well inside the radius where the potential changes from that of the galaxy to that of the black hole. Nonetheless, the dynamics of the accreting gas changes from that of a cooling flow to that of a Bondi flow at, or even outside of, $r_{\mathrm{tr}}$. The requirement that the flow undergo a smooth sonic transition influences its structure out to radii $\gtrsim r_{\operatorname{tr}} \gg r_{s}$.

The primary assumption of our analysis is that there is negligible angular momentum in the inflowing material. Angular momentum may, however, become important on the small scales of interest here; if so, a transition to a Bondi flow will not be viable. In this case, we believe that the cooling flow will generally undergo a transition to an advection-dominated accretion flow (ADAF), the hot accretion flow analogue of Bondi's solution for flows with angular momentum (Narayan \& Yi 1994, 1995; Abramowicz et al. 1995). It is well known that ADAFs can only exist below a critical accretion rate, $\dot{M}_{c}$. In the present context, this can be estimated by requiring the cooling time at $r_{\mathrm{tr}}$ to be greater than the inflow time, which yields

$$
\dot{M}_{c} \sim 10 \alpha^{2} M_{\odot} \mathrm{yr}^{-1}\left(\frac{M}{10^{9} M_{\odot}}\right)\left(\frac{\sigma}{300 \mathrm{~km} \mathrm{~s}^{-1}}\right)
$$

In equation (14), $\alpha$ is the ratio of the inflow velocity to the local Keplerian speed, and is roughly the Shakura \& Sunyaev (1973) viscosity parameter.

The properties of the transition from a cooling flow to a Bondi flow described in $\S 3$ are determined primarily by the low radiative efficiency of the Bondi solution. Consequently, the qualitative picture outlined in this paper should apply equally well to the transition from a cooling flow to an ADAF, since the latter is also radiatively inefficient (the quantitative details may, of course, change). If, however, the accretion rate supplied by the cooling flow to $r_{\text {tr }}$ is $\gtrsim \dot{M}_{c}$, the cooling flow may not be able to evolve into an ADAF; it will instead likely collapse to a thin disk. We plan to explore this question in a subsequent paper.

\subsection{Observational Prospects}

To conclude, we discuss the observational prospects for observing the transition from a cooling flow to an accretion flow with the excellent ( $\lesssim 1 \mathrm{arcsec}$ ) angular resolution of the CXO. We focus our discussion on M87 in the Virgo cluster, as it is the closest large cooling flow. For a distance to $\mathrm{M} 87$ of $\approx 18 \mathrm{Mpc}$, one arcsec is $\approx 0.1 \mathrm{kpc}$. The basic inference from Figures $1 \& 3$ is that on scales of $0.1 \mathrm{kpc}$, the dynamics of accreting gas in M87 is far from that of a cooling flow. The ratio of the cooling time to the inflow time is likely $\approx 10$ or larger.

One prediction of our model is that the inferred gas temperature in M87 should increase with decreasing radius on sufficiently small scales (when the black hole's potential dominates). In particular, all of our models show an increasing temperature profile for $r \lesssim 300$ pc. Note however, that because the gas temperature in nearly all models is roughly virial (and therefore traces the potential), such a temperature increase would not necessarily confirm the cooling flow to accretion flow transition. It would merely show that the gas dynamics is dominated by a centrally condensed mass distribution (namely, the black hole).

The second prediction of our model is that the X-ray surface brightness profile, while centrally peaked, should be suppressed with respect to that expected from a cooling flow without a central point mass. This is because the

\footnotetext{
${ }^{3}$ M87's surface brightness profile is observed to flatten around $r_{b} \approx 0.7 \mathrm{kpc}$ (Faber et al. 1995).
} 
transition from a cooling flow to an accretion flow entails a decrease in radiative efficiency. A suppressed X-ray surface brightness profile is, however, also the characteristic signature of a cooling flow with mass drop out.

At a minimum, our analysis indicates that significant care must be taken in interpreting the X-ray surface brightness profiles of elliptical galaxies on $\lesssim 1 \mathrm{kpc}$ scales. Standard analysis techniques, based on the assumption of a cooling flow, could incorrectly infer a radially decreasing accretion rate when in fact the accretion is via a Bondi flow with constant $\dot{M}$ and it is the radiative efficiency that decreases. As shown in $\S 3$ (see Figs. $2 \& 5$ ), $\dot{M}$ is expected to be nearly constant on the small scales of interest here since the transition to a Bondi-like flow strongly suppresses mass drop out due to thermal instability.

Given an observed X-ray luminosity $L_{X}$ and sound speed $c_{s}$, the true local accretion of the flow is

$$
\dot{M} \approx \frac{L_{X}}{c_{s}^{2}} \frac{t_{\mathrm{cool}}}{t_{\mathrm{inf}}} .
$$

Our calculations indicate that $t_{\text {cool }} / t_{\text {inf }}$ is likely to be $\gtrsim 10$ on $\approx 0.1 \mathrm{kpc}$ scales in M87 so that accretion rates inferred assuming a cooling flow $\left(t_{\text {cool }} \approx t_{\text {inf }}, \dot{M} \approx L_{X} / c_{s}^{2}\right)$ can be underestimates by up to an order of magnitude. This is shown explicitly in Figure 5 where we compare the true accretion rate for our baseline $(q=0.6)$ model of M87 (solid line) with that which we would infer if we took the predicted X-ray surface brightness and temperature profiles and assumed a pure cooling flow (dotted line).

X-ray spectra may break the degeneracy between a change in radiative efficiency and mass drop out. The presence of co-spatial multi-temperature components in coolings flows is indicated by a detailed examination of their Xray spectral lines (Canizares et al. 1979, 1982; Mushotzky et al. 1981). This is interpreted as evidence for mass drop out in the cooling flow. Consequently, the absence of evidence for such multi-temperature components (or a decrease in their prominence), together with a suppression in the X-ray surface brightness at small radii, would provide considerable support for our model of the cooling flow to accretion flow transition.

We acknowledge support from an NSF Graduate Research Fellowship (EQ) and NSF Grant 9820686 (RN). We thank the referee for useful comments which significantly improved this paper.

\section{REFERENCES}

\author{
Abramowicz, M., Chen, X., Kato, S., Lasota, J.-P., \& Regev, O., \\ 1995, ApJ, 438, L37 \\ Bailey, M.E., 1980, MNRAS, 191, 195 \\ Bondi, H., 1952, MNRAS, 112, 19 \\ Canizares, C.R. et al., 1979, ApJ, 234, L33 \\ Canizares, C.R., Clark, G.W., Jernigan, J.G.,\& Markert, T.H., \\ 1982, ApJ, 262, 33 \\ Cowie, L. L. \& Binney, J., 1977, ApJ, 215, 723 \\ Faber, S.M. et al. 1997, AJ, 114, 1771 \\ Fabian, A. C., 1994, ARA\&A, 32, 277 \\ Fabian, A. C. \& Nulsen, P.E.J., 1977, MNRAS, 180, 479 \\ Ford, H. C. et al., 1994, ApJ, 435, L27 \\ Harms, R.J. et al. 1994, ApJ, 435, L35 \\ Kormendy, J. \& Richstone, D., 1995, ARA\&A, 33, 581 \\ Lauer, T. R. et al., 1995, AJ, 110, 2622
}

Macchetto, F., Marconi, A., Axon, D.J., Capetti, A., Sparks, W., \& Crane, P., 1997, ApJ, 489, 579

Matthews, W.G. \& Bregman, J.N., 1978, ApJ, 224, 308

Mushotzky, R.F., Holt, S.S., Smith, B.W., Boldt, E.A., \& Serlemitsos, P.J., 1981, ApJ, 244, L47

Narayan, R., \& Yi, I., 1994, ApJ, 428, L13

Narayan, R., \& Yi, I., 1995, ApJ, 452, 710

Nulsen, P.E.J., Stewart, G.C., \& Fabian, A.C., 1984, 208, 185

Paczyński, B. \& Wiita, P. J., 1980, A\&A, 88, 23

Sarazin, C.L. \& White, R.E., 1987, ApJ, 320, 32

Shakura, N. I., \& Sunyaev, R. A., 1973, A\&A, 24, 337

Stewart, G.C., Canizares, C.R., Fabian, A.C., \& Nulsen, P.E.J., 1984, ApJ, 278, 536

White, R. E. \& Sarazin, C. L., 1987, ApJ, 318, 612 

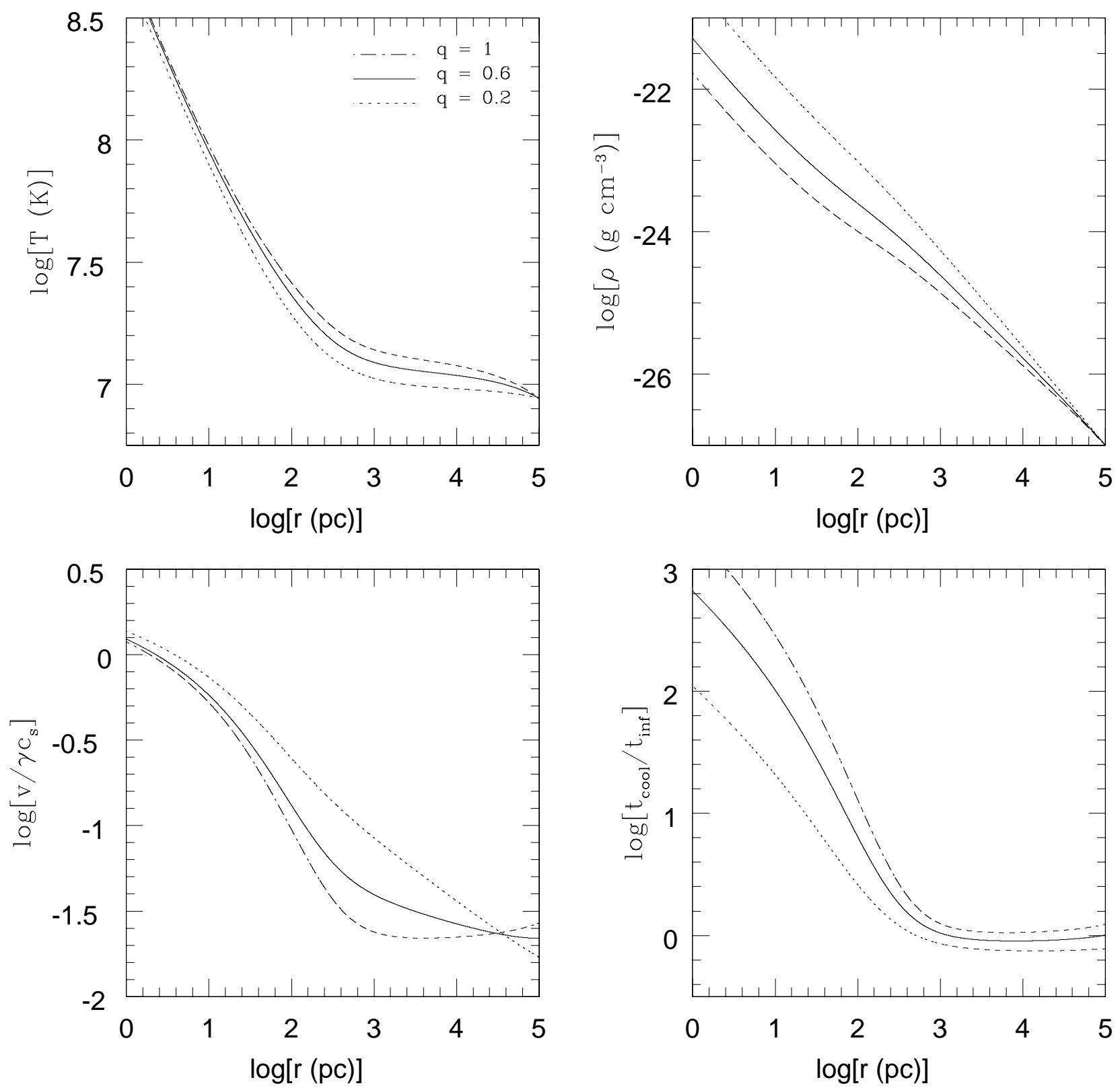

Fig. 1.- Numerical models of the transition from a cooling flow to an accretion flow. The temperature, density, ratio of cooling time to inflow time, and Mach number are shown (clockwise, from top left). The outer boundary conditions are chosen to represent M87. The $q=0.6$ model reproduces M87's radially varying $\dot{M}$ reasonably well (Fig. 2). Models for two additional values of $q$ are shown to indicate the range of expected behavior. 


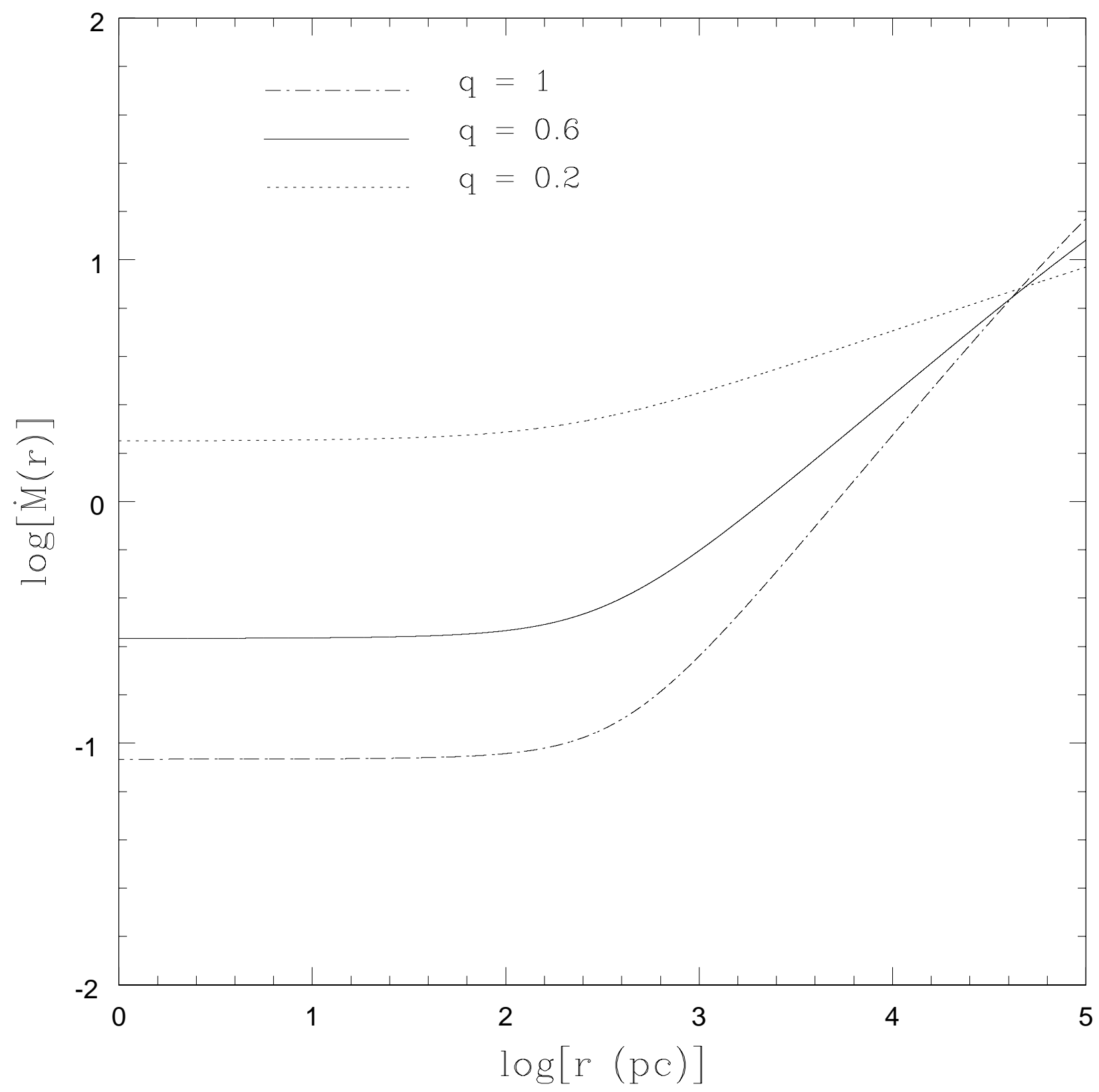

Fig. 2. - The accretion rate as a function of radius for the three models whose structure is shown in Figure 1 . The $q=0.6$ model roughly represents M87, for which observations show a decrease in $\dot{M}$ from $\approx 10 M_{\odot} \mathrm{yr}^{-1}$ at $\approx 70 \mathrm{kpc}$ to $<1 M_{\odot} \mathrm{yr}^{-1}$ at a few kilo-parsecs. The presence of a central black hole (taken to have $M=3 \times 10^{9}$ ) and the associated transition to a nearly adiabatic accretion flow leads to a complete suppression of mass drop out on small scales. This accounts for the constant accretion rate on scales less than $\approx 300$ parsecs. 

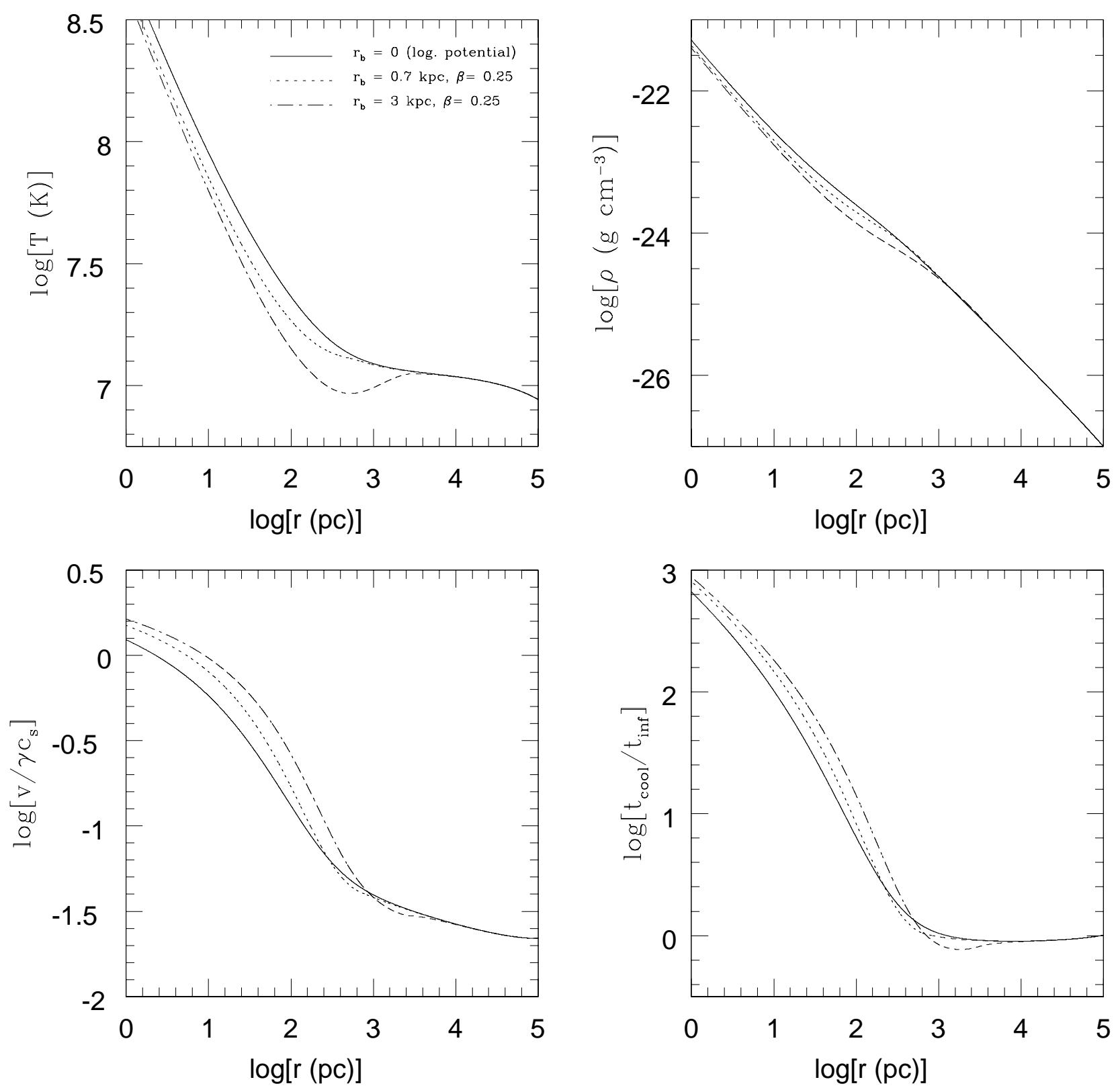

Fig. 3.- Numerical models of the cooling flow to accretion flow transition for three possible underlying potentials for the host galaxy (see eqs. [4] and [5]). The outer boundary conditions are chosen to represent M87. The temperature, density, ratio of cooling time to inflow time, and Mach number are shown (clockwise, from top left). 


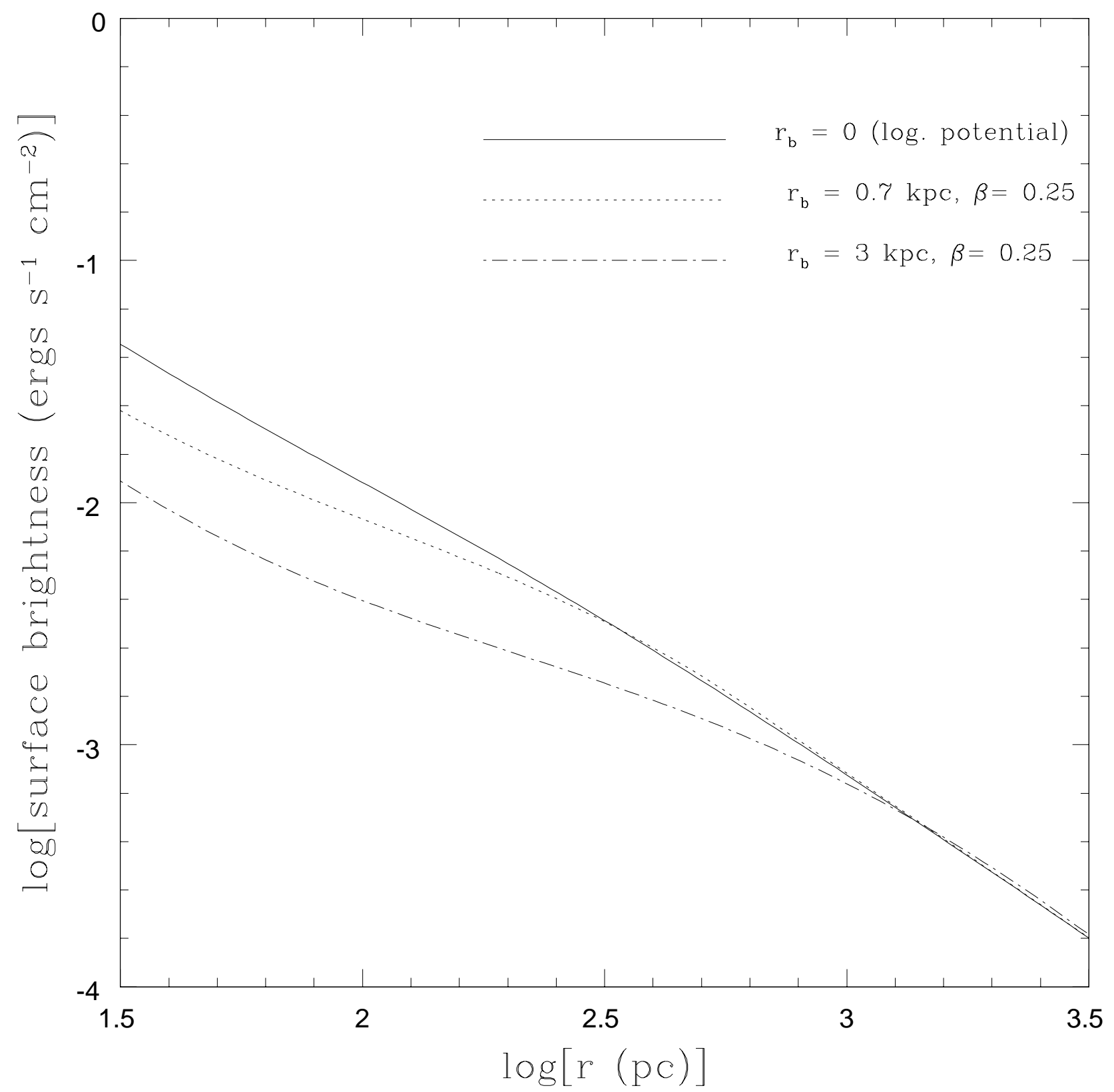

Fig. 4.- Predicted X-ray surface brightness profiles for the three models whose structure is shown in Figure 3. 


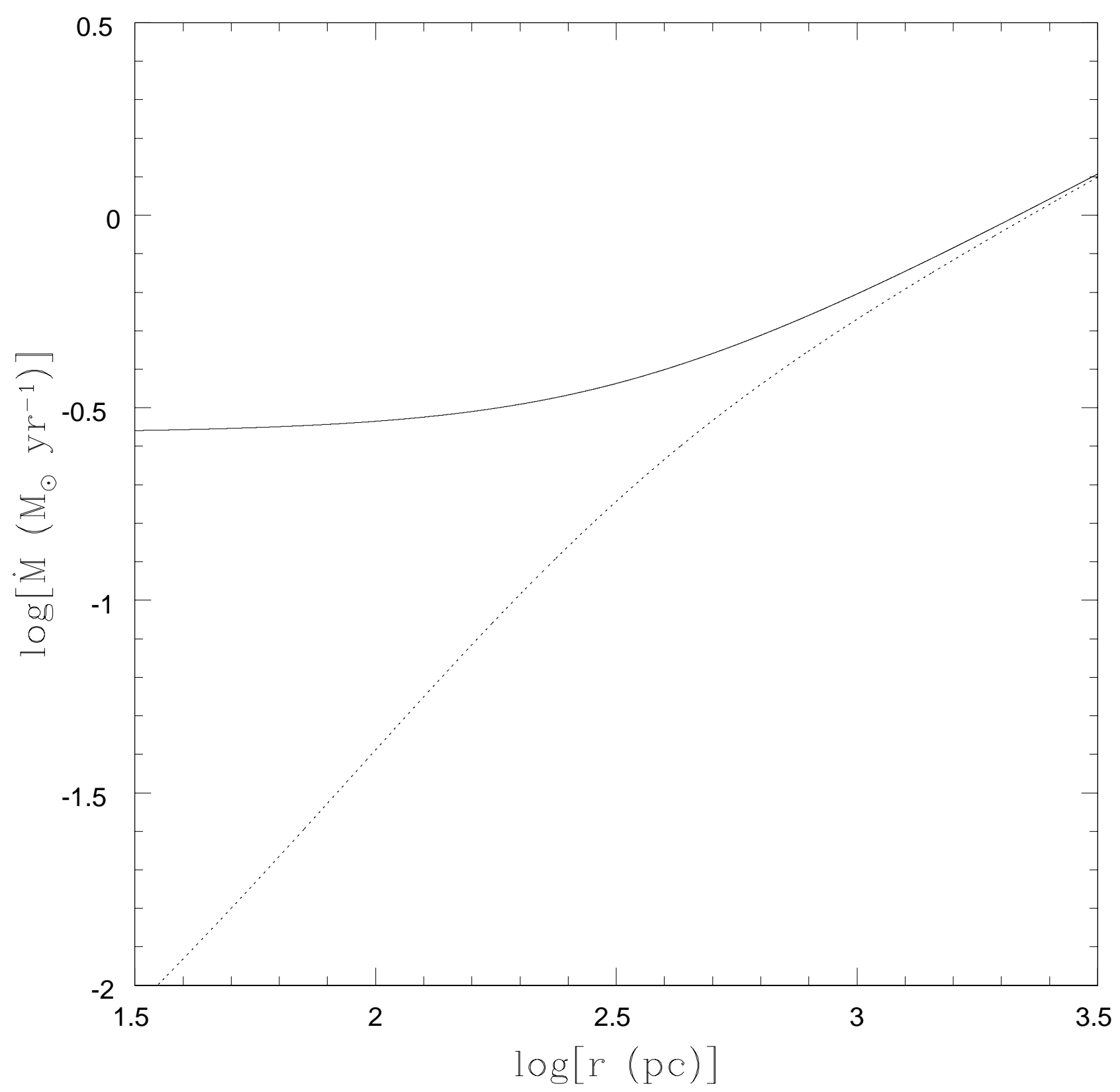

Fig. 5. - The solid line shows the true radially varying accretion rate for our $q=0.6$ model of M87 (pure logarithmic potential; solid line in Figs. 3 \& 4). The dotted line shows the accretion rate inferred from the predicted X-ray surface brightness and temperature profiles if one assumes that the gas is a pure cooling flow. The true accretion rate is nearly constant on the small scales shown in this figure because the transition from a cooling flow to a nearly adiabatic accretion flow suppresses mass drop out due to thermal instability. The decreasing radiative efficiency associated with this transition could, however, be misinterpreted as a radially decreasing accretion rate (dotted line). 